\title{
Prevalência de Síndrome Metabólica em uma área rural de Santa Rosa
}

\author{
Prevalence of metabolic syndrome in a rural area of Santa Rosa \\ Prevalencia del síndrome metabólico en una zona rural de Santa Rosa \\ Roger Silveira Haab*, Luís Antônio Benvegnú ${ }^{2}$, Ediléia Venske Fischer ${ }^{3}$
}

Palavras-chave: Síndrome X Metabólica

Epidemiologia

Obesidade Abdominal Atenção Primária à Saúde
Keywords:

Metabolic Syndrome X

Epidemiology

Abdominal Obesity

Primary Health Care

\section{Resumo}

Síndrome Metabólica (SM) é uma condição de prevalência elevada e crescente. Este artigo tem como objetivo descrever os determinantes e também a prevalência deste importante fator de risco para doenças cardiovasculares em uma área rural do município de Santa Rosa. Foi realizado um estudo transversal, em uma população de abrangência da Estratégia Saúde da Família, constituída de adultos jovens na faixa etária de 20 a 49 anos. Foi aplicado um questionário pré-codificado com variáveis sociodemográficas, comportamentais, histórico pessoal e familiar de doenças crônicas, bem como obtidas medidas antropométricas e laboratoriais pertinentes. Para a classificação da Síndrome Metabólica, esta pesquisa seguiu os critérios da National Cholesterol Education Program's Adult Treatment Panel (NCEP/ATP III) e da International Diabetes Federation. Foram descritos componentes isolados da SM, encontrando alta prevalência de obesidade abdominal e HDL - colesterol baixo em mulheres, bem como alta prevalência de níveis pressóricos aumentados em homens. A prevalência global da SM encontrada foi de 8,5\% em homens e 22\% em mulheres pelos critérios da NCEP/ATP III, e 15,9\% em homens e 25,3\% em mulheres pelos critérios da IDF.

\section{Abstract}

Metabolic Syndrome (MS) is a condition of high prevalence and growing. This article aims to describe the determinants and also the prevalence of this important risk factor for cardiovascular disease in a rural area of the Santa Rosa city. We conducted a cross-sectional study in a population covered by the Family Health Strategy, consisting of young adults aged 20-49 years. We applied a pre-coded questionnaire with socio-demographic, behavioral, personal and family history of chronic diseases, and obtained anthropometric measurements and laboratory tests. For the classification of the metabolic syndrome, this study followed the criteria of the National Cholesterol Education Program's Adult Treatment Panel (NCEP/ATP III) and the International Diabetes Federation. We described a single component of MS and found a high prevalence of abdominal obesity and low $\mathrm{HDL}$ - cholesterol in women and high prevalence of increased blood pressure in men. The overall prevalence of MS was found to be $8.5 \%$ in men and $22 \%$ in women by the criteria of the NCEP/ATP III, and $15.9 \%$ in men and $25.3 \%$ in women by IDF criteria.

\footnotetext{
${ }^{1}$ Fundação Municipal de Saúde de Santa Rosa (FUMSSAR). rs.haab@terra.com.br

2 Fundação Municipal de Saúde de Santa Rosa (FUMSSAR). labenvegnu@brturbo.com.br

${ }^{3}$ Fundação Municipal de Saúde de Santa Rosa (FUMSSAR). ev.fischer@terra.com.br

*Autor correspondente.

Fonte de financiamento: Fundação Municipal de Saúde de Santa Rosa (FUMSSAR) e Núcleo de Ensino e Pesquisa de Santa Rosa (NEP).

Conflito de interesses: declaram não haver.

Recebido em: 27/09/2011

Aprovado em: 14/04/2012
} 


\begin{abstract}
Palabras clave: Resumen
Síndrome Metabólico X

Epidemiología

La Obesidad Abdominal

Atención Primaria de Salud

El síndrome metabólico (SM) es una condición de alta y creciente prevalencia. Este artículo pretende describir los factores determinantes y también laprevalencia de este importante factor de riesgopara enfermedad cardiovascular en una zona rural del municipio de Santa Rosa. Se realizó unestudio transversal en una población que abarca la Estrategia de Salud Familiar, que consiste enlos adultos jóvenes de 20-49 años. Se aplicó un cuestionario pre-codificado con la historiasocio-demográficos, de comportamiento,personal y familiar de las enfermedades crónicas,y obtuvieron medidas antropométricas y de laboratorio pertinentes. Para la clasificación del síndrome metabólico, este estudio dio seguimiento a los criterios del Panel del National Cholesterol Education Program Adult Treatment (NCEP/ATP III) y la Federación Internacional de Diabetes. Hemos descrito un único componente de la MS y se encontró una alta prevalencia de obesidad abdominal y niveles bajos de HDL - colesterol en las mujeres y la alta prevalencia de presión arterial elevada en los hombres. La prevalencia global de el síndrome metabólico se encontró que era del 8,5\% en hombres y $22 \%$ en las mujeres por parte de los criterios de la III del NCEP/ATP, y el $15,9 \%$ en hombres y25,3\% en las mujeres por parte de la IDF.
\end{abstract}

\section{Introdução}

A Síndrome Metabólica (SM) é representada por um transtorno complexo. Caracteriza-se por um conjunto de fatores de risco metabólico e cardiovascular, comumente relacionados à obesidade, principalmente à obesidade abdominal e à resistência periférica à insulina. Deve ser destacada a importância da SM do ponto de vista epidemiológico, visto aumentar a mortalidade por doenças cardiovasculares em 2,5 vezes ${ }^{1}$.

Reaven $^{2}$, já na década de 1980 observou que a dislipidemia, a hipertensão arterial e a hiperglicemia eram condiçóes frequentemente encontradas em um mesmo indivíduo e estas conferiam maior risco cardiovascular, situação à qual denominou síndrome X. Frequentemente, outras alteraçóes metabólicas estão presentes nesses indivíduos, tais como aumento do fator ativador plasmático do fibrinogênio e do plasminogênio, hiperuricemia, aumento da Proteína $\mathrm{C}$ Reativa, hiper-homocisteinemia, aumento da expressão do TNF-alfa, e diminuição dos níveis de adiponectina².

O rápido crescimento da ocorrência dessa condição nas últimas décadas tem sido atribuído às mudanças principalmente de origem demográfica, tais como urbanização, industrialização e envelhecimento das populaçóes, bem como a alteraçôes do estilo de vida, como hábitos dietéticos inadequados, sedentarismo e estresse, sendo que, níveis de atividade física têm impacto em todos os principais componentes da $\mathrm{SM}^{3}$. Da mesma forma, dietas ricas em açúcares simples e em amido refinado e pobre em fibras, frutas, verduras, legumes, grãos integrais, nozes e leguminosas também podem ter papel importante no seu desenvolvimento ${ }^{4}$.

A síndrome é uma condição de prevalência elevada e crescente em algumas populaçóes, como por exemplo, as afrodescendentes, méxico-americanas e hispânicas ${ }^{3}$. Nos Estados Unidos, a prevalência é semelhante em homens e mulheres, e aumenta de maneira importante com a idade, passando de 7\% na faixa de 20-29 anos até 43\% naqueles com 60 anos ou mais ${ }^{4}$.
O estudo da SM tem sido dificultado pela ausência de consenso na sua definição e nos pontos de corte dos seus componentes, com repercussões na prática clínica e nas políticas de saúde. No Brasil, não foram encontrados estudos sobre a prevalência de $\mathrm{SM}$ com dados representativos da populaçáo brasileira ${ }^{1}$. É importante conhecermos nossa realidade para o adequado direcionamento das açóes de saúde, sobretudo para o estabelecimento de medidas de prevenção primária e secundária, visto serem altos os custos socioeconômicos decorrentes da morbimortalidade cardiovascular ${ }^{3}$.

A definição de SM do National Cholesterol Education Program's Adult Treatment Panel III - (NCEP-ATP III $)^{4}$ foi desenvolvida para uso clínico e não exige a comprovaçáo da resistência à insulina, facilitando a sua utilização. Sendo assim, pela sua simplicidade, é a definição recomendada pela I Diretriz Brasileira de Diagnóstico e Tratamento da Síndrome Metabólica (I - DBSM)ํ․ Já a International Diabetes Federation (IDF) centralizou o diagnóstico de SM na obesidade central, sendo esta essencial, seguida de mais dois componentes 5 .

O objetivo deste estudo foi estudar a prevalência de SM e descrever a frequência de seus componentes na populaçáo "adulto-jovem" de uma área rural de Santa Rosa. Os resultados ajudarão as equipes a mobilizar os habitantes, de modo a modificar tal situação com medidas preventivas dentro e fora da Atenção Primária à Saúde.

\section{Material e métodos}

Conduziu-se um estudo epidemiológico observacional, descritivo, do tipo transversal, cuja base populacional se constituiu dos moradores de uma área rural de Santa Rosa, na abrangência da Estratégia Saúde da Família, com 100\% de cobertura de agentes comunitários. Santa Rosa contém 68.221 habitantes, conforme o censo do IBGE, $2010^{6}$, vivendo 
em área rural, e sua economia gira em torno da agricultura familiar, principalmente pela cultura da soja, milho, trigo, suinocultura e produçáo de vaca leiteira. As origens étnicas predominantes na regiáo são a alemá, a italiana e a polonesa.

Calculou-se o tamanho da amostra utilizando-se o programa Epi Info 2000 v.3.5.17, com base na prevalência de síndrome metabólica estimada em $20 \%$ para a população de 20 a 49 anos, com margem de erro de 6\%, que resultou numa amostra necessária de 208 indivíduos, já incluído 30\% para fatores de confusão e $10 \%$ para perdas. A seleção foi realizada através de uma relação dos moradores obtida no Sistema de Informação da Atenção Básica $(\mathrm{SIAB})^{8}$. Foi feita uma lista ordenada por sexo e idade, resultando num total de 982 indivíduos. A partir de então, foi feita uma amostragem sistemática com pulo de quatro indivíduos, sendo que, nos primeiros quatro números, foi sorteado um, (iniciado aleatoriamente), resultando numa listagem de 245 indivíduos.

Foram elegíveis indivíduos com 20 a 49 anos, homens e mulheres, com exceção das grávidas ou nos dois primeiros meses pós-parto, concordantes em assinar o Termo de Consentimento Livre e Esclarecido (TCLE). Os critérios de exclusão foram: 1) evidências de tireoidopatias, doenças renais ou hepáticas, estabelecidas pelo histórico pessoal de doenças crônicas, coletado durante o questionário pré-codificado; 2) uso de medicamentos que acabam interferindo na homeostase glicêmica, exceto hipoglicemiantes orais, (e.g. corticosteroides de uso crônico); e 3) incapacidade psicomotora para realização da coleta de dados. Foram constatadas 69 perdas $(28,2 \%)$, porque os moradores não foram encontrados ou recusaram-se a participar da pesquisa. Foram excluídos 3 indivíduos (1,4\%), 2 devido a tireoidopatia e 1 devido a gestação.

Entre junho e novembro de 2010, os indivíduos selecionados, entrevistados por pessoal previamente treinado, foram chamados ao Posto de Saúde de Guia Lopes, da Estratégia Saúde da Família local. Na ocasião foi aplicado um questionário com perguntas fechadas, pré-codificado, abordando componentes demográficos como idade, sexo, renda, se possuía ou não propriedade rural, situação conjugal atual, anos de escolaridade. Também foram abordados aspectos comportamentais como sedentarismo, uso de bebida alcoólica, coletado através do questionário CAGE, sendo positivo com uma resposta afirmativa e tabagismo. Foi considerado hiperglicemia valores glicêmicos iguais ou maiores que $110 \mathrm{mg} / \mathrm{mL}$, conforme proposto pela NCEP/ATP III, ou uso de hipoglicemiantes, e diabetes quando referia diagnóstico médico ou quando fazia uso de hipoglicemiantes. Pressão arterial elevada foi considerada quando os pacientes apresentaram PAS $>$ ou $=130 \mathrm{mmHg}$ e/ou PAD $>$ ou $=85 \mathrm{mmHg}$, ou faziam uso de anti-hipertensivos com PA normal. Avaliou-se história familiar através do conhecimento do entrevistado de presença de hipertensão, diabetes, cardiopatia e dislipidemia, ou uso de medicamentos para estas doenças em pai, mãe ou irmãos. A história pessoal de hipertensão, diabetes, cardiopatia, doenças renais, hepáticas e tireoidopatias foi autorreferida.

A pressão arterial foi medida pela técnica auscultatória conforme recomendam as V Diretrizes Brasileiras de Hipertensão Arterial', utilizando esfigmomanômetro aneroide, calibrado, revisado anualmente pelo INMETRO. O manguito foi adaptado no braço esquerdo do sujeito e o estetoscópio posto na topografia da artéria braquial. A aferição da pressão arterial foi realizada após repouso de 5 minutos, estando o entrevistado com a bexiga vazia, sentado, com os pés apoiados ao chão e calmo. Foi verificado entáo o peso, a altura, a circunferência abdominal e a circunferência do quadril com fita métrica não elástica e, então, após 2 minutos, foi aferida novamente a pressão arterial. A medida utilizada na pesquisa foi a média das duas aferiçóes.

O peso e a altura serviram para o cálculo do IMC, através da fórmula: $I M C=$ peso $(\mathrm{kg}) /$ altura $(\mathrm{m})^{2[10]}$. O diâmetro da cintura realizou-se com o paciente em pé, circundando-a na altura da cicatriz umbilical ${ }^{3}$ e o diâmetro do quadril, circundando-o na protuberância maior dos glúteos ${ }^{11}$. Os indivíduos foram pesados sem sapatos, sem casacos e sem acessórios.

Para a obtenção da amostra de sangue por punção venosa, os indivíduos foram encaminhados ao laboratório municipal da Fundação Municipal de Saúde (FUMSSAR). Os exames foram realizados em data posterior à aplicação do questionário, em um dia estabelecido por acordo mútuo, em que o indivíduo foi orientado a estar em jejum de 12 horas. Desde a punção venosa até o resultado final, os exames foram feitos por técnicos do laboratório. O sangue foi centrifugado a $3.500 \mathrm{rpm}$ por 3 minutos a fim da obtenção da amostra (soro). Os exames realizados foram colesterol total, colesterol HDL, triglicerídeos e glicemia de jejum pelo método enzimático colorimétrico, em um aparelho de bioquímica BTS 370 plus automatizado. Os exames foram processados no mesmo dia da realização da coleta.

Para o diagnóstico de Síndrome Metabólica, foram utilizados os parâmetros convencionados pela I Diretriz Brasileira de Diagnóstico e Tratamento da Síndrome Metabólica ${ }^{1}$, a qual adotou o que foi estabelecido pela National Cholesterol Education Program's Adult Treatment Panel III (NCEP/ATPIII) $)^{4}$, segundo a qual a combinação de três dos cinco componentes a seguir concluem o diagnóstico: 1 - glicemia de jejum maior ou igual a $110 \mathrm{mg} / \mathrm{dL}$ ou uso de hipoglicemiante; 2- PAS maior ou igual a $130 \mathrm{mmHg}$ ou PAD maior ou igual a $85 \mathrm{mmHg}$, ou uso de anti-hipertensivos; 3 -HDL colesterol menor que $40 \mathrm{mg} / \mathrm{dL}$ para homens e menor 
que $50 \mathrm{mg} / \mathrm{dL}$ para mulheres; 4 - triglicerídeos maior ou igual a $150 \mathrm{mg} / \mathrm{dL}$; 5- diâmetro da cintura maior que $102 \mathrm{~cm}$ para homens e maior que $88 \mathrm{~cm}$ para mulheres.

Foram utilizados neste estudo também os critérios do International Diabetes Federation (IDF) ${ }^{5}$, para SM. Para tanto, a circunferência abdominal normal da mulher ficou estipulada em até $80 \mathrm{~cm}$ e do homem latino-americano em $90 \mathrm{~cm}$. Os demais componentes equiparam-se à definiçáo do NCEP-ATP III ${ }^{4}$.

Para o diagnóstico de Obesidade, utilizou-se o IMC, com os seguintes pontos de corte: menor que $20,0 \mathrm{~kg} / \mathrm{m}^{2}$ - baixo peso; 20 a $24,9 \mathrm{Kg} / \mathrm{m}^{2}$ - peso normal; 25,0 a $29,9 \mathrm{~kg} / \mathrm{m}^{2}$ - sobrepeso; mais que $30 \mathrm{~kg} / \mathrm{m}^{2}$ - obesidade.

A obesidade abdominal foi estabelecida com base no diâmetro da cintura, segundo os critérios do NCEP, acima de $102 \mathrm{~cm}$ em homens e acima de $88 \mathrm{~cm}$ para mulheres, e também segundo critérios da IDF, maior que $90 \mathrm{~cm}$ em homens e acima de $80 \mathrm{~cm}$ em mulheres. Foi utilizado, alternativamente, para o cálculo da obesidade central, a relaçáo cintura/quadril (RCQ), com os pontos de corte estipulados pela Organização Mundial da Saúde (OMS) ${ }^{4}$ : para homens, maior que 0,90 e para mulheres maior que 0,85 .

Para o diagnóstico de dislipidemias, os valores de referência corresponderam aos critérios das III Diretrizes sobre Dislipidemias ${ }^{12}$ : hipercolesterolemia com colesterol total igual ou maior que $240 \mathrm{mg} / \mathrm{dL}$; hipertrigliceridemia, quando triglicerídeos foram maior que 200; HDL- colesterol baixo, quando menor que $40 \mathrm{mg} / \mathrm{dL}$.

Se a glicemia de jejum fosse maior que $126 \mathrm{mg} / \mathrm{dL}$ em indivíduos que desconheciam ser diabéticos, seria proposto uma consulta subsequente, orientando o indivíduo a uma nova coleta de glicemia em 30 dias para confirmar ou descartar o diagnóstico. Isto não ocorreu em nossa pesquisa.

Os dados foram digitados em dupla digitação no programa EPI INFO, e em seguida foi realizada comparaçáo e limpeza dos dados. A análise consistiu de obtenção da construção de variáveis a serem estudadas e obtençâo de suas medidas de tendência e distribuição na população e estratificação por sexo e idade. Para comparação dos resultados nos estratos, utilizou-se geralmente o $\mathbf{x}^{2}$ e o valor $\mathrm{p}$.

Este estudo foi aprovado pelo Comitê de Ética em Pesquisa em Seres Humanos da Universidade Regional do Noroeste do Estado do Rio Grande do Sul - UNIJUÍ, pelo parecer consubstanciado $\mathrm{n}^{\circ}$ 069/2010 de acordo com a resoluçáo n 196/96 do Conselho Nacional de Saúde. Todos os participantes foram informados dos objetivos do projeto, concordaram e assinaram o Termo de Consentimento Livre e Esclarecido. Os indivíduos com exames alterados foram orientados a procurar o responsável pela pesquisa, e realizar acompanhamento médico subsequente.

\section{Resultados}

Foram efetivamente entrevistados e tiveram exames realizados 173 sujeitos. A amostra foi constituída de 82 homens $(47,4 \%)$ e 91 mulheres $(52,6 \%)$. A média de idade foi de 35,2 anos $(\mathrm{dp} 8,6)$ para homens e 34,3 anos (dp 8,8) para mulheres $(\mathrm{p}=0,51)$ (Tabela 1$)$.

A situação conjugal, tanto dos homens quanto das mulheres, demonstrou que a grande maioria, $79,3 \%$ dos homens e $79,1 \%$ das mulheres, vivia com companheiro $(\mathrm{p}=0,98)$. A renda familiar foi relativamente baixa, sendo a média salarial mensal de 2,5 salários mínimos. No entanto, apenas $2,9 \%$ ganhavam menos que um salário mínimo. Os homens ganhavam mais quando a renda foi superior a 2,5 salários mínimos. A renda das mulheres foi mais baixa, predominando a abaixo de 2,5 salários mínimos $(\mathrm{p}=0,12)$.

Com relação à escolaridade, $59,8 \%$ dos homens e $58,2 \%$ das mulheres estudaram até 8 anos, revelando uma média de escolaridade de 8,3 anos ( $\mathrm{dp} 3,55)$ para homens e 8,0 (dp 3,53) para mulheres, denotando uma população de baixa escolaridade, na qual a maioria não completou o ensino básico.

Predominam os não fumantes, $82,9 \%$ dos homens e $94,5 \%$ das mulheres $(p=0,02)$. Os tabagistas homens fumam em média 15,7 cigarros/dia e as mulheres 12,0 cigarros/dia

Tabela 1. Distribuição das características sociodemográficas em adultos de área rural na faixa etária de 20 a 49 anos, Santa Rosa - RS, 2010.

\begin{tabular}{|c|c|c|c|c|c|}
\hline \multirow{2}{*}{ Variáveis } & \multicolumn{2}{|c|}{ Sexo masculino } & \multicolumn{2}{|c|}{ Sexo feminino } & \multirow{2}{*}{ *P } \\
\hline & $N=82$ & $\%$ & $\mathrm{~N}=91$ & $\%$ & \\
\hline Idade (anos) & & & & & 0,51 \\
\hline $20-29$ anos & 26 & 31,7 & 30 & 33,0 & \\
\hline $30-39$ anos & 24 & 29,3 & 27 & 29,7 & \\
\hline $40-49$ anos & 32 & 39,0 & 34 & 37,4 & \\
\hline Média (dp) & & $35,2(8,6)$ & & $34,3(8,8)$ & \\
\hline Situação conjugal & & & & & 0,98 \\
\hline Vive com companheiro & 65 & 79,3 & 72 & 79,1 & \\
\hline Não vive com companheiro & 17 & 20,7 & 19 & 20,9 & \\
\hline Renda (salário mínimo) & & & & & 0,12 \\
\hline Até $1,5 \mathrm{sm}$ & 16 & 19,5 & 23 & 25,3 & \\
\hline $1,5-2,5 \mathrm{sm}$ & 17 & 20,7 & 26 & 28,6 & \\
\hline $2,5-3,5 \mathrm{sm}$ & 20 & 24,4 & 19 & 20,9 & \\
\hline$>3,5 \mathrm{sm}$ & 29 & 35,4 & 23 & 25,3 & \\
\hline Escolaridade & & & & & 0,66 \\
\hline Até 8 anos & 49 & 59,8 & 53 & 58,2 & \\
\hline $9-11$ anos & 22 & 26,8 & 30 & 33,0 & \\
\hline 12 anos ou mais & 11 & 13,4 & 8 & 8,8 & \\
\hline
\end{tabular}

${ }^{*} \mathrm{P}=$ valor $\mathrm{p}$ 
( $p=0,48)$; e os homens fumam em média há 16,5 anos e as mulheres há 15,6 anos $(p=0,85)$. Um décimo da amostra admitiu consumir álcool pelo menos uma vez por dia. A exposição ao álcool é bem maior nos homens $26,8 \%$, sendo de 6,6\% em mulheres ( $\mathrm{p}<0,001$ )(Tabela 2).

Com relação à atividade física, as mulheres são mais sedentárias, $67 \%$ não realizam nenhuma atividade física. Uma pequena parcela da população realiza atividade física com frequência, cinco vezes por semana, no mínimo 30 minutos. Uma parcela importante dos que realizam alguma atividade física não praticam desta forma, principalmente indivíduos do sexo masculino $(\mathrm{p}=0,03)$.

A prevalência de sobrepeso entre os homens foi de $45,1 \%$ e para mulheres $28,6 \%$; e na obesidade, os homens participam com $12,2 \%$ e as mulheres com $28,6 \%$, constatando uma população em sua maioria com excesso de peso $57,2 \%(p=0,18)$. A prevalência da RCQ aumentada é muito importante, sendo de $46,3 \%$ para homens e de $82,4 \%$ em mulheres $(\mathrm{p}=0,33)$.

Ao descrevermos os componentes isolados da SM, estratificados por sexo e faixa etária, obtivemos resultados em que a obesidade abdominal, segundo os critérios da NCEP, evidenciou-se importante, especialmente nas mulheres. Nos homens, foi mais expressiva a partir de 40 anos. As mulheres apresentaram uma maior prevalência de obesidade abdominal $(64,8 \%)$ em relação aos homens $(17,1 \%)$, revelando taxas mais altas em faixa etária mais jovem e progredindo com a idade. Quando a classificação é baseada nos critérios da IDF, a obesidade abdominal alcança níveis mais elevados, $58,5 \%$ para homens e $85,7 \%$ para mulheres (Tabela 3 ).

Em relação à pressão arterial, os homens apresentaram maior prevalência de pressão arterial elevada $(51,2 \%)$, comparando às mulheres $(26,4 \%)(p<0,01)$. No que refere ao baixo HDL, a prevalência deste perfil dislipidêmico foi mais acentuado no sexo feminino, em todas as faixas etárias $(53,8 \%)$, sendo nos homens mais baixa - $(11,9 \%)(\mathrm{p}<0,01)$. $\mathrm{O}$ que não se observou nas taxas de triglicerídeos, que foi mais predominante no sexo masculino $(24,4 \%)(\mathrm{p}=0,09)$.

Levando em conta a glicemia de jejum, as mulheres apresentaram ligeira predominância de hiperglicemia $(3,3 \%)$, em relação aos homens $(1,2 \%)$, sem significância estatística $(p=0,36)$. Esta hiperglicemia correspondeu em sua totalidade a casos de DM tipo 2 encontrados, sendo que nas duas faixas etárias mais jovens, de ambos os sexos, não se obteve casos de alteração do perfil glicêmico.

Ao ser realizada a pesquisa sobre os antecedentes familiares de doenças crônicas não transmissíveis, bem como à morbidade pessoal referida, as mulheres apresentaram maior prevalência de antecedentes familiares, bem como referiram morbidade pessoal com mais frequência. As mulheres faziam, também, mais uso crônico de medicamentos do que os homens (Tabela 4).
Tabela 2. Distribuição das características sociocomportamentais em adultos, de zona rural, na faixa etária de 20 - 49, Santa Rosa - RS, 2010.

\begin{tabular}{|c|c|c|c|c|c|}
\hline \multirow{2}{*}{ Variáveis } & \multicolumn{2}{|c|}{ Sexo masculino } & \multicolumn{2}{|c|}{ Sexo feminino } & \multirow{2}{*}{  } \\
\hline & $\mathrm{N}=82$ & $\%$ & $\mathrm{~N}=91$ & $\%$ & \\
\hline Tabagismo & & & & & 0,02 \\
\hline Não fumantes & 68 & 82,9 & 86 & 94,5 & \\
\hline Fumantes & 14 & 17,1 & 5 & 5,5 & \\
\hline$N^{\circ}$ de cigarros dia & & 15,7 & & 12,0 & 0,48 \\
\hline Média ( $d p)$ & & $(11,1)$ & & $(4,5)$ & \\
\hline Tempo de tabagismo & & 16,5 & & 15,6 & 0,85 \\
\hline anos (dp) & & $(9,3)$ & & $(7,3)$ & \\
\hline \multicolumn{6}{|l|}{ Consumo de álcool } \\
\hline${ }^{*} \mathrm{CAGE}=1$ ou mais & 22 & 26,8 & 6 & 6,6 & $\begin{array}{c}< \\
0,001\end{array}$ \\
\hline Atividade física & & & & & 0,03 \\
\hline Sedentários & 42 & 51,2 & 61 & 67,0 & \\
\hline $\begin{array}{c}5 \text { vezes por semana, } \\
30 \text { minutos }\end{array}$ & 5 & 6,1 & 12 & 13,2 & \\
\hline $\begin{array}{c}\text { Menos } 5 \text { vezes por } \\
\text { semana }\end{array}$ & 35 & 42,7 & 18 & 19,8 & \\
\hline${ }^{\star *} \mid \mathrm{MC}$ & & & & & 0,18 \\
\hline$<20$ & 4 & 4,9 & 5 & 5,5 & \\
\hline 20 a 24,9 & 31 & 37,8 & 34 & 37,4 & \\
\hline 25 a 29,9 & 37 & 45,1 & 26 & 28,6 & \\
\hline 30 ou mais & 10 & 12,2 & 26 & 28,6 & \\
\hline \multicolumn{6}{|l|}{${ }^{\star * \star} \mathrm{RCQ}$ alterada } \\
\hline (OMS) & 43 & 52,4 & 75 & 82,4 & 0,33 \\
\hline
\end{tabular}

${ }^{*}$ CAGE = questionário CAGE para consumo de álcool. ${ }^{*} \mid \mathrm{MC}=$ índice de massa corporal $\mathrm{dp}$ - desvio padrão. ${ }^{\star \star \star} \mathrm{R} C Q$ razão cintura quadril alterada. ${ }^{\star \star \star \star} \mathrm{P}=$ valor $\mathrm{p}$.

A SM, segundo a NCEP, teve uma prevalência global de $15,6 \%$ ( $8,5 \%$ para homens e $22 \%$ para mulheres), e segundo a IDF, de 20,8\% (15,9\% para homens e 25,3\% para mulheres). Ao descrever a prevalência da SM, estratificados por sexo e faixa etária, observamos que as mulheres têm maior prevalência de SM do que os homens, dentro dos critérios do NCEP e da IDF, em todas as faixas etárias (Tabela 5).

A prevalência de SM pelo critério da NCEP em homens aumenta de forma importante a partir dos 40 anos, passando de $4,1 \%$ na faixa de 30 a 39 anos para $15,6 \%$ na faixa de 40 a 49 anos. Com as mulheres, isto ocorre em estágio mais precoce, a partir dos 30 anos, assumindo uma prevalência de $6,6 \%$ na faixa de 20 a 29 anos para $29,6 \%$ na faixa de 30 a 39 anos. Segundo os critérios da IDF, que valoriza principalmente a obesidade abdominal, a prevalência em homens, com mais de 40 anos foi de $25 \%$. Já entre as mulheres a partir de 30 anos, a prevalência foi de 33,3\%. 
Tabela 3. Descrição dos componentes isolados da SM, estratificados por sexo e faixa etária, em zona rural de Santa Rosa - RS, 2010.

\begin{tabular}{|c|c|c|c|c|c|c|c|c|c|c|c|c|}
\hline \multirow{3}{*}{$\begin{array}{c}\text { Variáveis } \\
\text { Faixa etária (anos) }\end{array}$} & \multicolumn{6}{|c|}{ Sexo masculino } & \multicolumn{6}{|c|}{ Sexo feminino } \\
\hline & \multicolumn{2}{|c|}{$(20-29)$} & \multicolumn{2}{|c|}{$(30-39)$} & \multicolumn{2}{|c|}{$(40-49)$} & \multicolumn{2}{|c|}{$(20-29)$} & \multicolumn{2}{|c|}{$(30-39)$} & \multicolumn{2}{|c|}{$(40-49)$} \\
\hline & ${ }^{*} \mathrm{~N}$ & $\%$ & ${ }^{*} \mathrm{~N}$ & $\%$ & ${ }^{*} \mathrm{~N}$ & $\%$ & ${ }^{*} \mathrm{~N}$ & $\%$ & ${ }^{*} \mathrm{~N}$ & $\%$ & ${ }^{*} \mathrm{~N}$ & $\%$ \\
\hline & 26 & & 24 & & 32 & & 30 & & 27 & & 34 & \\
\hline${ }^{\star \star} 0 \mathrm{~A}-{ }^{* \star \star} \mathrm{NCEP}$ & 3 & 11,5 & 2 & 8,3 & 9 & 28,1 & 15 & 50 & 18 & 66,6 & 26 & 76,4 \\
\hline${ }^{\star \star} 0 \mathrm{~A}-{ }^{\star \star \star \star \star} \mid \mathrm{DF}$ & 12 & 46,1 & 12 & 50,0 & 24 & 75,0 & 22 & 73,0 & 24 & 88,8 & 32 & 94,1 \\
\hline PA elevada & 14 & 53,4 & 9 & 37,5 & 19 & 59,3 & 2 & 6,6 & 9 & 33,3 & 13 & 38,2 \\
\hline HDL baixo & 5 & 19,2 & 1 & 4,1 & 4 & 12,5 & 14 & 46,6 & 19 & 70,3 & 16 & 47,0 \\
\hline Triglicerídeos altos & 6 & 23,0 & 6 & 25,0 & 8 & 25,0 & 2 & 6,6 & 5 & 18,5 & 6 & 17,6 \\
\hline Hiperglicemia & 0 & 0 & 0 & 0 & 1 & 3,1 & 0 & 0 & 0 & 0 & 3 & 8,8 \\
\hline
\end{tabular}

${ }^{\star} \mathrm{N}=$ número. ${ }^{\star \star} \mathrm{OA}$ - obesidade abdominal. ${ }^{\star \star \star}$ NCEP - National Cholesterol Education Program's Adult Treatment Panel. ${ }^{\star \star \star \star} \mid \mathrm{DF}$ - International Diabetes Federation.

Tabela 4. Antecedentes Familiares de Doenças Crônicas e Morbidade Pessoal Referida, estratificados por sexo em população de 20-49 anos, Santa Rosa - RS, 2010.

\begin{tabular}{|c|c|c|c|c|c|}
\hline \multirow[t]{2}{*}{ Variáveis } & \multicolumn{2}{|c|}{ Sexo masculino } & \multicolumn{2}{|c|}{ Sexo feminino } & \multirow[b]{2}{*}{${ }^{*} \mathrm{P}$} \\
\hline & $\mathrm{N}=82$ & $\%$ & $\mathrm{~N}=91$ & $\%$ & \\
\hline \multicolumn{6}{|c|}{ Antecedentes familiares } \\
\hline D M & 14 & 17,1 & 29 & 31,9 & 0,27 \\
\hline HAS & 50 & 61,0 & 62 & 68,1 & 0,45 \\
\hline Cardiopatia & 28 & 34,1 & 45 & 49,5 & 0,63 \\
\hline Dislipidemia & 27 & 32,9 & 36 & 39,6 & 0,38 \\
\hline \multicolumn{6}{|c|}{ Morbidade pessoal referida } \\
\hline Diabetes & 1 & 1,2 & 2 & 2,2 & 0,20 \\
\hline HAS & 10 & 12,2 & 14 & 15,4 & 0,63 \\
\hline Cardiopatia & 3 & 3,7 & 6 & 6,6 & 0,97 \\
\hline \multicolumn{6}{|c|}{ Uso crônico de medicamentos } \\
\hline & 15 & 18,3 & 27 & 29,7 & 0,08 \\
\hline
\end{tabular}

${ }^{*} \mathrm{P}=$ valor $\mathrm{p}$.

Tabela 5. Prevalência de Síndrome Metabólica, segundo critérios NCEP/ ATP III e IDF, estratificados por sexo e faixa etária, zona rural de Santa Rosa - RS, 2010.

\begin{tabular}{ccccrc}
\hline Variáveis & N & Prev.Ncep $(\%)$ & P & Prev.ldf $(\%)$ & P \\
\hline Homens & 82 & $7(8,5)$ & 0,01 & $13(15,9)$ & 0,12 \\
20 a 29 anos & 26 & $1(3,8)$ & 0,19 & $3(11,5)$ & 0,19 \\
30 a 39 anos & 24 & $1(4,1)$ & & $2(8,3)$ & \\
40 a 49 anos & 32 & $5(15,6)$ & & $8(25,0)$ & \\
Mulheres & 91 & $20(22)$ & 0,01 & $23(25,3)$ & 0,12 \\
20 a 29 anos & 30 & $2(6,6)$ & 0,04 & $3(10,0)$ & 0,06 \\
30 a 39 anos & 27 & $8(29,6)$ & & $9(33,3)$ & \\
40 a 49 anos & 34 & $10(29,4)$ & & $11(32,3)$ & \\
$\quad$ Total & 173 & $27(15,6)$ & 0,01 & $36(20,8)$ & 0,12 \\
\hline
\end{tabular}

Prev.: prevalência. $P=$ valor $p . N=$ número.

\section{Discussão}

A prevalência de SM encontrada em nosso estudo foi elevada, aumentou com a idade e foi maior no sexo feminino. Dentre os componentes isolados, observamos que a obesidade abdominal e o HDL baixo foram mais expressivos nas mulheres, enquanto que os níveis pressóricos aumentados predominaram nos homens, como corroboram outros estudos ${ }^{3,11}$.

Nosso estudo apresentou elevado índice de perdas. É possível que entre os moradores que aceitaram participar do estudo houvesse um maior número de pacientes cadastrados no Posto de Saúde, resultando numa prevalência superestimada da doença em estudo. Da mesma forma, as perdas diminuem o poder estatístico do estudo em observar diferenças moderadas e pequenas, por isso, os resultados devem ser avaliados com cautela, principalmente aqueles que apresentaram diferenças sem significância estatística.

Um estudo na cidade de Vitória ${ }^{11}$ encontrou prevalência total de SM de 29,8\% (IC 95\% 28-32), porém a faixa etária estudada foi de 25-64 anos, com um incremento da prevalência nas faixas etárias mais altas 45-64 anos. De Sá et al. ${ }^{13}$, em Brasília, encontraram prevalência semelhante a nossa em faixas etárias mais jovens, $5,9 \%$ na faixa de 25 a 34 anos e $12 \%$ na faixa etária de 35 a 44 anos. Outro estudo realizado em zona 
rural do semiárido baiano ${ }^{3}$ em 2005 , encontrou prevalência por idade, de $7 \%$ na faixa de 25 a 34 anos e $21,9 \%$ na faixa de 35 a 44 anos, ocorrendo elevaçáo desta taxa ao se considerar idade maior que 45 anos. Por outro lado, estudo realizado em zona rural de Minas Gerais, encontrou prevalência menor do que o nosso estudo entre os homens $(7,7 \%)$, e prevalência maior entre as mulheres $(33,6 \%)^{14}$.

É importante observarmos que a SM já começa em adolescentes. Uma revisão sistemática com 16 estudos na faixa de 12 a 19 anos, encontrou taxas de 2,2\% a 15,3\% segundo os critérios da NCEP, o que reforça múltiplos fatores ambientais e genéticos envolvidos, principalmente a obesidade ${ }^{15}$.

Ao avaliarmos a situação conjugal, percebemos que a grande maioria da população em nosso estudo teve união estável. Aqueles que viviam com companheiro, em ambos os sexos, tiveram média de perímetro abdominal significativamente maior do que os demais, mas mais expressivamente em homens, como mostra em um estudo avaliando variáveis sociodemográficas e gordura abdominal, no sul do Brasil ${ }^{16}$. Porém a união conjugal estável, em um estudo avaliando fatores associados à SM, no Distrito Federal, náo foi significativa como fator de risco para o desenvolvimento da doença ${ }^{13}$.

A renda é fator que tem impacto nas áreas de lazer, nos níveis de atividade física, no acesso a uma alimentação mais saudável, e está relacionada com a escolaridade ${ }^{13}$. Em um estudo avaliando indicadores de obesidade centralizada, o nível socioeconômico mais baixo, (interaçáo renda/escolaridade), idade, sexo feminino e sedentarismo mantiveram associaçâo estatisticamente significativa com elevação da razão cintura/quadril(RCQ) ${ }^{17}$. Outros trabalhos também relacionaram menor renda e obesidade abdominal ${ }^{11,18}$. Por outro lado, em um estudo no sul do Brasil, alto nível socioeconômico esteve associado significativamente à obesidade abdominal ${ }^{16}$. Em nosso estudo, as mulheres tiveram menor renda, foram mais obesas, assim como a grande maioria teve alteração da RCQ e da circunferência abdominal. As diferenças no desenvolvimento da SM entre os sexos em relação ao nível socioeconômico requerem estudos adicionais que demandam conhecimento de como variam os componentes individuais da SM nas diferentes classes sociais ${ }^{11}$.

Em um trabalho realizado em Cotia, o tabagismo apresentou efeito protetor na obesidade centralizada ${ }^{17}$. O ex-tabagismo, porém, demonstrou estar relacionado a maiores médias de perímetro abdominal, segundo estudo feito em Pelotas ${ }^{16}$. A exposição ao álcool é mais prevalente em homens e não mostrou associação significativa com a obesidade abdominal nestes estudos ${ }^{16,17}$. A interação tabagismo/etilismo não se associou significativamente com a RCQ, existindo até um efeito protetor em relação à circunferência abdominal ${ }^{17}$. Em nosso estudo, a prevalência de tabagismo e alcoolismo foi baixa, porém maior em homens, sendo que a obesidade abdominal não foi expressiva neste estrato.
Em nosso estudo, foi baixa a prevalência dos indivíduos que praticavam atividade física com regularidade. Apenas 6,1\% dos homens e 13,2\% das mulheres realizavam alguma atividade física cinco vezes por semana, no mínimo 30 minutos. O efeito do exercício físico sobre a sensibilidade à insulina tem sido demonstrado de 12 a 48 horas após a sessão de exercício, voltando aos níveis anteriores em três a cinco dias de inatividade, o que reforça a necessidade de praticar atividade física com frequência e regularidade ${ }^{19}$. Os benefícios do exercício regular também se estendem para os diabéticos, hipertensos, dislipidêmicos e obesos, devendo a sua prescrição ser orientada segundo os princípios da sobrecarga, especificidade, individualidade e reversibilidade. Portanto, os achados do nosso estudo indicam a necessidade de a Equipe Saúde da Família (ESF) trabalhar a atividade física como proposta para a manutenção da saúde e prevenção de uma grande variedade de doenças crônicas, além da $S M^{19}$.

No Brasil, os resultados da Pesquisa de Orçamentos Familiares (POF- IBGE) ${ }^{20}$ realizada em 2008-09, em parceria com o Ministério da Saúde, evidencia que o peso dos brasileiros vem aumentando nos últimos anos, com diminuição importante dos indicadores de desnutrição infantil. O excesso de peso já começa na infância, passando pela adolescência e repercutindo na fase adulta em todos os grupos de renda. Destaca-se, nesta pesquisa, a Região Sul, que apresenta os maiores percentuais de obesidade. Deste modo, a influência do aumento da expectativa de vida, somado ao estilo de vida predominantemente urbano, mesmo em zonas rurais, devido ao acesso a bens de consumo que diminuem o esforço físico, e o fácil acesso à indústria alimentícia, podem estar implicados no ganho de peso corporal. Segundo as recomendaçóes das III Diretrizes Brasileiras sobre Dislipidemias ${ }^{12}$, o excesso de peso - IMC $>25 \mathrm{~kg} / \mathrm{m}^{2}$, principalmente o acúmulo de gordura na região abdominal, está associado a um maior risco de doença aterosclerótica. $\mathrm{O}$ mesmo estudo enfatizou que a Mudança do Estilo de Vida (MEV), que leve à perda de peso, como exercícios, dieta e, em casos específicos, farmacoterapia, é de extrema importância. Ainda mais, a maioria das análises econômicas e dos estudos de custo-efetividade sobre o manejo da dislipidemia concentra-se nos custos associados com doenças cardiovasculares ${ }^{12}$.

Percebe-se que mais da metade da população estudada teve excesso de peso, mas uma obesidade bem mais expressiva nas mulheres, com taxa de $28,6 \%$ para IMC acima de $30 \mathrm{Kg} / \mathrm{m}^{2}$, assim como maior prevalência de $\mathrm{SM}$ em mulheres. Conforme estudo avaliando risco cardiovascular em população de obesos, foi encontrada nítida relação entre aumento do IMC e prevalência de HAS e correlação positiva entre obesidade e diabetes ${ }^{21}$. Em outro estudo realizado em Campos ${ }^{22}$, a prevalência de hipertensão, diabetes e dislipidemias, esteve diretamente relacionada à IMC > 30 e à circunferência 
abdominal aumentada, concluindo que quanto maior a obesidade, maior é a prevalência dos componentes da SM, assumindo um perfil de risco desfavorável para doença cardiovascular.

O excesso de peso encontrado na maioria de nossa população parece indicar que a ingestáo calórica de alimentos excede o gasto energético. A alimentação inadequada aliada ao sedentarismo ou à prática insuficiente de atividade física da grande maioria da populaçáo gera acúmulo de calorias, elevando sua taxa metabólica basal ${ }^{4}$. É importante observarmos isto na Atenção Primária à Saúde, enfocando a obesidade como um problema de saúde interdisciplinar, abordando inclusive suas raízes socioculturais e familiares.

Com relação à obesidade abdominal, os mecanismos fisiopatogênicos pelos quais a gordura visceral pode associar-se a resistência à insulina e à $\mathrm{SM}$ são muito pesquisados. $\mathrm{O}$ comportamento metabólico da gordura intra-abdominal difere do tecido adiposo subcutâneo periférico ou gluteofemoral, sendo o primeiro mais sujeito à lipólise, expressa maior número de receptores de corticoides e é mais sensível à adrenalina e à noradrenalina. Acredita-se que os ácidos graxos livres provenientes da lipólise da gordura visceral, liberados em grande quantidade na circulação portal, tenham papel definitivo na gênese da resistência tecidual à ação da insulina. Além disto, outros fatores acentuam a resistência à insulina, como a atividade inflamatória subclínica. Estudos revelam que a redução da circunferência da cintura correlacionou-se com a diminuiçáo dos valores da proteína $\mathrm{C}$ reativa ${ }^{23}$.

Acredita-se que a obesidade abdominal em mulheres esteja relacionada também à fase adulta e às gestações. Um estudo realizado na cidade do Rio de Janeiro apontou que a idade e paridade estiveram associadas à obesidade central em mulheres em idade reprodutiva, mas que a maior escolaridade, significou fator de proteção para elevaçáo da razão cintura/quadril ${ }^{24}$. A prevalência de obesidade abdominal em homens é menor do que nas mulheres, o que já foi demonstrado em vários estudos ${ }^{3,5,11,14}$. Um estudo em Pelotas observou que a idade, baixa renda e união estável estavam estatisticamente associadas com a obesidade abdominal em homens ${ }^{18}$. Em nosso estudo, a obesidade abdominal foi demonstrada pela alteração RCQ e pelo aumento da circunferência abdominal. Dentre os fatores envolvidos, podemos constatar que a obesidade centralizada esteve relacionada com a idade, porém com intensidade bem maior nas mulheres. Esta faixa etária compreende o período reprodutivo da mulher, o que pode ter implicado a sua história gestacional. Também é preciso levar em conta a possibilidade de algum viés de seleção que, em função das perdas, teria entrevistado um número maior de obesas por terem maior vínculo com o serviço de saúde. No entanto, a maioria das mulheres tem escolaridade baixa ou é sedentária, o que poderia estar implicando a gênese da alta prevalência da obesidade abdominal encontrada no sexo feminino.
Outro componente da SM estudado foi a elevação da pressão arterial. Os níveis pressóricos elevados foram mais prevalentes em homens. O sexo masculino e a idade representam fatores de risco para HAS, como foi demonstrado em um estudo realizado em Goiânia, onde também o excesso de peso e circunferência da cintura estiveram significativamente associados ${ }^{25}$. A relevância da HAS como importante fator de risco cardiovascular, sua alta prevalência mundial, torna muito importante o conhecimento de sua relação com outros possíveis fatores desencadeantes de desfechos cardiocirculatórios. A prevalência de pressão arterial elevada encontrada foi alta, ao contrário do encontrado na literatura ${ }^{9,25,26}$, pois, em nosso estudo, utilizamos o ponto de corte $130 \mathrm{X} 85 \mathrm{mmHg}$, enquanto os estudos citados utilizaram $160 \mathrm{X} 95 \mathrm{mmHg}$ ou 140 X $90 \mathrm{mmHg}$. Jardim ${ }^{25}$ encontrou índice global de HAS de $36,4 \%$, enquanto Passos $^{26}$ relatou prevalência de $24,7 \%$ em homens e de 19,8\% em mulheres em um artigo de revisão. Passos $^{26}$ avalia que a HAS no Brasil é influenciada por fatores etários, econômicos e sociais, estando sua ocorrência associada a outros fatores de risco para doenças cardiovasculares, como IMC e dislipidemia, sendo grande a variabilidade das informaçôes obtidas em função de diversos fatores, como desenho do estudo, população e abrangência geográfica. Outro aspecto que deve ser lembrado é a possibilidade de viés de seleção, como mencionado em relação à obesidade, em que os indivíduos que aceitaram participar da pesquisa eram os que já tinham vínculo com a unidade em função da própria doença. Neste caso, o aumento do número de pessoas com pressão arterial elevada e obesas levaria também a um aumento da estimativa de Síndrome Metabólica encontrada em nosso estudo. A HAS já vem sendo abordada intensamente na ESF local, através de tratamento não medicamentoso e farmacológico. É necessário sempre insistir no potencial benefício destas intervençóes, garantindo seu acompanhamento através da integralidade, da coordenação e da longitudinalidade ${ }^{27}$.

O componente HDL colesterol baixo descrito em nosso estudo, revelou maior prevalência em mulheres. Isto pode ser reflexo do sedentarismo, predominante nesta população. Porém não podemos deixar de reconhecer que os critérios NCEP para SM são mais rígidos para o sexo feminino. Os triglicerídeos altos foram mais prevalentes nos homens, mas não de modo expressivo, o que poderia ser reflexo da maior exposição ao álcool desta parcela da população. Em um estudo realizado em nipo-brasileiros, os níveis de HDL - colesterol baixo - também foram mais prevalentes em mulheres e os triglicerídeos mais prevalentes em homens, mas de modo mais expressivo, devido a esta população ter nível elevado de DM tipo $2^{28}$.

A prevalência de DM tipo 2 foi de 3,1\% nos homens acima de 40 anos e 8,8\% nas mulheres acima de 40 anos, sendo que nas faixas etárias mais jovens não ocorreram casos. 
Pode se observar que nesta faixa etária a obesidade abdominal foi mais prevalente nos dois sexos, sendo mais expressiva nas mulheres. Em um estudo avaliando o perfil epidemiológico de pacientes diabéticos cadastrados no HiperDia ${ }^{29}$ de Cuiabá, a prevalência aumentou com a idade, principalmente acima de 40 anos, foi predominante no sexo feminino, em pessoas que tiveram o ensino fundamental incompleto e naqueles que conviviam com companheiro. Os diabéticos apresentavam circunferência da cintura aumentada e tinham sobrepeso ou obesidade ${ }^{30}$.

A SM parece ser transmitida hereditariamente, mas a atual pandemia se deve não tanto à carga genética, mas muito mais à mudança de expressão gênica resultante do confronto de nossos genes com os fatores ambientais e o estilo de vida diferentes daqueles presentes na origem de nossa espécie ${ }^{4}$. Sem dúvida, o peso corporal do adulto e em particular a massa de tecido adiposo é o resultado da interação entre os fatores genéticos e um ambiente favorável à utilização e armazenamento de energia consumida, sem ter que lutar pela sobrevivência em períodos de escassez, como o faziam nossos ancestrais ${ }^{31}$. Nesse estudo, as mulheres apresentaram maior prevalência de antecedentes mórbidos familiares e também pessoais de doenças crônicas não transmissíveis, mas não de modo significativo. Porém estudos específicos são necessários para pesquisar a transmissão genética da resistência à insulina, cuja prevalência é de $45 \%$ em parentes de primeiro grau de pacientes com diabetes tipo $2^{4}$. Outros aspectos a serem considerados são os ligados a fatores culturais, familiares e aos hábitos do cotidiano, que podem influenciar para que os casais e/ou familiares apresentem HAS, DM ou obesidade, situação em que a atuação do Médico de Família e Comunidade pode ter grande impacto na atuaçáo preventiva e educativa, manejando as situaçóes que determinam a presença dos fatores de risco e das morbidades citadas.

A amostra de sangue foi processada no mesmo dia da coleta e os dados foram digitados duplamente, para correçáo de possíveis erros. Isto contribuiu para o estudo alcançar boa confiabilidade dos dados e das informaçōes, conferindo acurácia para análise e confecção do relatório que, certamente, auxiliará na tomada das decisóes de planejamento e gestấo da saúde no município.

\section{Conclusão}

A SM é, portanto, uma combinação de elementos, que contribuem para mortalidade mediante desfechos cardiovasculares. Estes desfechos são consequência de um processo iniciado muito antes, talvez na infância. A história natural da SM pode modificar-se com açôes que mudem o curso clínico das condições que determinam sua incidência.
Dentre estas condiçóes se encontram o excesso de peso, o sedentarismo e a dieta inadequada ${ }^{32}$.

A prevalência da SM encontrada em nosso estudo justifica ações para a sua prevenção, visto que afeta as pessoas em idades produtivas e podem representar custos elevados para a sociedade. Níveis pressóricos elevados foram predominantes nos homens. Obesidade abdominal e HDL colesterol baixo tiveram maior prevalência nas mulheres. A SM é um desafio para a APS, sendo que melhorias nas condiçôes socioeducativas são de primordial importância.

É improvável que uma intervenção isolada possa modificar esta situação. Uma nova realidade implica num processo educativo para entender a enfermidade, através de mudanças significativas, envolvendo uma equipe interdisciplinar (médicos de família e comunidade somados a outros especialistas médicos, além de nutricionistas, professores de educação física, enfermeiros, psicólogos, entre outros). Deve existir uma relaçáo intersetorial, mas principalmente uma aliança terapêutica com a família e a comunidade, pois é muito importante para a mudança do estilo de vida. Somam-se também a estes, fatores culturais, próprios de cada regiáo que devem ser compreendidos para melhorar a adesão ao tratamento.

Desta forma, a realizaçáo deste estudo permitiu observar, em pacientes que estáo no cadastro ambulatorial da comunidade de Guia Lopes e aceitaram entrar no estudo, uma prevalência de SM de $8,5 \%$ em homens e $22 \%$ em mulheres. Foi encontrada maior prevalência de níveis pressóricos elevados em homens e uma importante história familiar de hipertensão, diabetes e cardiopatia. A alta prevalência de obesidade abdominal nas mulheres foi o fator mais importante para a prevalência de SM observada, o que deve direcionar o tratamento. Porém se fazem necessários outros estudos analisando os fatores de risco envolvidos, a fim de implementar programas educativos apropriados que evitem seu desenvolvimento.

\section{Referências}

1. Sociedade Brasileira de Cardiologia. I diretriz brasileira de diagnóstico e tratamento de síndrome metabólica. Arq Bras de Cardiol. 2005;(1): 3-28.

2. Lottenberg SA, Glezer A, Turatti LA. Metabolic syndrome: identifying the risk factors. J Pediatr. 2007; (5): 204-8.

3. De Oliveira EP, De Souza MLA, De Lima MDA. Prevalência de síndrome metabólica em uma área rural do semi-árido baiano. Arq Bras Endocrinol Metab. 2006; (3): 456-65.

4. Duncan BB. Medicina ambulatorial: condutas de atenção primária baseada em evidências. Porto Alegre: Artmed; 2004.1600 p.

5. Nakazone MA. Prevalência de síndrome metabólica em indivíduos brasileiros pelos critérios NCEP-ATP III e IDF. Rev 
Assoc Med Bras. 2007; 53(5):407-13. http://dx.doi.org/10.1590/ S0104-42302007000500016

6. Instituto Brasileiro de Geografia e Estatística - IBGE. Apresenta dados sobre população urbana e rural das cidades brasileiras [online]. Disponível em: www.ibge.gov.br/cidadesat/topwindow.htm?1.

7. Dean AG, Arner TG, Sunki GG, Friedman R, Lantinga M, Sangam $S$, et al. Epi Info, a database and statistics program for public health professionals. Atlanta: Center for Disease Control and Prevention; 2007.

8. Brasil. Ministério da Saúde. Sistema de Informação de Atenção Básica. Brasília; 1998. Manual desenvolvido pelo Ministério da Saúde, apresenta informações específicas do sistema. Disponível em: 189.28.128.100/dab/docs/publicações/geral/manual_siab2000. pdf.

9. Sociedade Brasileira de Cardiologia. V diretrizes brasileiras de hipertensão arterial. Arq Bras Cardiol. 2007; (3): 24-79.

10. Leite CC. Obesidade. In: Sociedade Brasileira de Clínica Médica - SBCM. Programa de atualização em Clínica Médica. Porto Alegre: ARTMED; 2004. p. 89-125.

11. Salaroli LB, Barbosa GC, Mill JG, Molina MCB. Prevalência de síndrome metabólica em estudo de base populacional, vitória, es-brasil. Arq Bras Endocrinol Metab. 2007; (7): 1143-52.

12. Sociedade Brasileira de Cardiologia. III diretrizes brasileiras sobre dislipidemias. Arq Bras Cardiol. 2001;(3): 1-48.

13. De Sá NNB, Moura EC. Fatores associados à carga de doenças da síndrome metabólica entre adultos brasileiros. Cad Saúde Pública. 2010; (9): 1853-62. PMid:20877945.

14. Meléndez GV, Gazzinelli A, Oliveira RC, Pimenta AM, Kac G. Prevalence of metabolic syndrome in a rural area of Brazil. São Paulo Med J. 2007; (3): 155-62. PMid:17923940.

15. De Moraes ACF, Fulaz CS, Oliveira ERN, Reichert FF. Prevalência de síndrome metabólica em adolescentes: uma revisão sistemática. Cad Saúde Pública. 2009; (6): 1195-1202. PMid:19503950.

16. Castanheira M, Olinto MTA, Gigante DP. Associação de variáveis sócio-demográficas e comportamentais com a gordura abdominal em adultos: estudo de base populacional no sul do Brasil. Cad Saúde Pública. 2003; (1): 55-65. http://dx.doi.org/10.1590/ S0102-311X2003000700007

17. Martins SI, Marinho SP. O potencial diagnóstico dos indicadores da obesidade centralizada. Rev Saúde Pública. 2003; (6): 760-7. http:// dx.doi.org/10.1590/S0034-89102003000600011

18. Olinto MTA, Nácul LC, Dias-da-Costa JS. Níveis de intervenção para obesidade abdominal: prevalência e fatores associados. Cad
Saúde Pública. 2006; (6): 1207-1215. http://dx.doi.org/10.1590/ S0102-311X2006000600010

19. Ciolac EG, Guimarães GV. Exercício físico e síndrome metabólica. Rev Bras Med Esporte. 2004; (4): 319-24.

20. Brasil. Ministério da Saúde. Pesquisa de orçamentos familiares. IBGE; 2008.

21. Cercato C, Silva S, Sato A, Mancini M, Halpern A. Risco cardiovascular em uma população de obesos. Arq Bras Endocrinol Metab. 2000; (1): $45-8$.

22. De Souza LJ, Gicovate Neto CG, Chalita FEB. Prevalência de obesidade e fatores de risco cardiovascular em campos, Rio de Janeiro. Arq Bras Endocrinol Metab. 2003; (6): 669-76.

23. Ribeiro Filho FF, Mariosa LS, Ferreira SRG. Gordura visceral e síndrome metabólica: mais que uma simples associação. Arq Bras Endocrinol Metab. 2006; (2): 230-38.

24. Kac G, Meléndez GV, Coelho MA. Fatores associados à obesidade abdominal em mulheres em idade reprodutiva. Rev Saúde Pública. 2001; (1): 46-51. http://dx.doi.org/10.1590/S0034-89102001000100007

25. Jardim PC, Gondim MR, Monego ET. Hipertensão arterial e alguns fatores de risco em uma capital brasileira. Arq Bras Cardiol. 2007; (4): 452-57. PMid:17546277. http://dx.doi.org/10.1590/ S0066-782X2007000400015

26. Passos VMA, Assis TD, Barreto SM. Hipertensão arterial no brasil: estimativa de prevalência a partir de estudos de base populacional. Epid Serv Saúde. 2006; 15(1): 35-45

27. Starfield B. Atenção primária: equilíbrio entre necessidades de saúde, serviços e tecnologia. Brasília: UNESCO; Ministério da Saúde; 2002. 726 p.

28. Siqueira AFA, Harima HA, Osiro K. Distúrbios no perfil lipídico são altamente prevalentes em população nipo-brasileira. Arq Bras Endocrinol Metab. 2008; (1): 40-46.

29. Brasil. Ministério da Saúde. Sistema de cadastramento e acompanhamento de hipertensos e diabéticos. Rio de Janeiro; 2002. Manual de Operação.

30. Ferreira CL, Ferreira MG. Características epidemiológicas de pacientes diabéticos da rede pública de saúde - análise a partir do sistema hiperdia. Arq Bras Endocrinol Metab.2009; (1): 80-86.

31. Schnell M, Dominguez ZA, Carrera C. Aspectos genéticos, clínicos y fisiopatológicos del síndrome metabólico. An Venez Nutr. 2007; (2): $92-98$

32. Villalobos JA, Meléndez JA, Esqueda AL. Las enfermedades crônicas no transmisibles em méxico: sinopsis epidemiológica y prevención integral. Salud Publica Mex. 2008; (5): 419-427. PMid:18852939. 